\title{
Exact Traveling Wave Solutions and Simulation For A Class Nonlinear Partial Differential Equations
}

\author{
Yan ping Ran \\ School of Mathematics and Statistics, Tianshui Normal University, Gansu \\ Tianshui 741001, China \\ shulixue@sina.com
}

\begin{abstract}
In the different subsets of 4-parameters space $\Omega=\left\{(k, r, c, \alpha) \mid(k, r, c, \alpha) \in R^{4}\right\}$, we obtain the exact traveling wave solutions of a class nonlinear partial differential equations which are closely related to the periodic wave solutions of $(3+1)$-dimensional nonlinear evolution equations, generated by the Jaulent-Miodek hierarchy. These wave solutions are verified by numerical simulation.
\end{abstract}

Keywords: Nonlinear $(3+1)$-dimensional equation, Traveling wave solution, Unbounded wave solutions.

\section{Introduction}

The third of $(3+1)$-dimensional nonlinear models generated by the Jaulent-Miodek hierarchy ${ }^{[1]}$, which is

$$
w_{t}=\frac{1}{4}\left(w_{x x}-2 w^{3}\right)_{x}-\frac{3}{4}\left(\frac{1}{4} \partial_{x}^{-1} w_{y y}+w_{x} \partial_{x}^{-1} w_{y}\right)+\alpha \partial_{x}^{-1} w_{z z}
$$

where $\alpha$ is a constant, $\partial_{x}^{-1}$ is the inverse of $\partial_{x}$ with $\partial_{x} \partial_{x}^{-1}=\partial_{x}^{-1} \partial_{x}=1$, and 


$$
\partial_{x}^{-1}=\int_{-\infty}^{x} f(t) d t
$$

Using the potential

$$
w(x, y, z, t)=u_{x}(x, y, z, t),
$$

to remove the integral term in (1.1), the system (1.1) becomes

$$
u_{x t}+\frac{1}{4} u_{x x x x}-\frac{3}{2} u_{x}^{2} u_{x x}+\frac{3}{16} u_{y y}+\frac{3}{4} u_{x x} u_{y}-\alpha u_{z z}=0 .
$$

The system (1.1) is a completely integrable evolution equations. There are many methods to be used in travel wave solutions of nonlinear evolution equations, such as the inverse scattering method, the B $\ddot{a}$ cklund transformation method, algebraic-geometric method, the Darboux transformation method, multiple exp-function method ${ }^{[4]}$, the Hirota bilinear method ${ }^{[2,3,8]}$, and dynamical systems method . By applying the dynamical systems method[5,6,7], some new exact solutions of (1.1) are obtained ${ }^{[7]}$. Since the structure of traveling wave solutions of (1.4) plays a large role in investigating the wave solutions of (1.1), we will study the solutions of traveling wave equations of (1.4) by the method of dynamical systems.

Let

$$
u(x, y, z, t)=\Psi(k x+r y+m z-c t)=\Psi(\xi),
$$

where $C$ is propagating wave velocity. Without loss of generality, let always assume that $(r, k, m, c, \alpha) \neq 0$.let $k \neq 0, m=1$, substituting (1.5) into the equation (1.4), we have ${ }^{[7]}$

$$
\frac{d \varphi}{d \xi}=y,
$$

$$
\frac{d y}{d \xi}=a \varphi+b \varphi^{2}+2 \varphi^{3}
$$

where $u_{\xi}=\varphi, a=-\frac{\left(3 r^{2}-16 k c-16 \alpha\right)}{4 k^{4}}, b=-\frac{3 r}{2 k^{2}}$.

The system (1.6) has the first integrate 


$$
H(\varphi, y)=9 y^{2}-9 a \varphi^{2}+6 b \varphi^{3}-9 \varphi^{4} .
$$

Multiple kink solutions, multiple singular kink solutions and multiple soliton solutions were formally derived ${ }^{[1]}$ and the exact traveling wave solutions of (1.1) have been obtained ${ }^{[7]}$.To our knowledge the study for the exact traveling wave solutions of (1.4) in different subsets of 4-parameters space of the system (1.7), has not been considered yet. By using the method of the dynamical systems, we shall generally investigate wave solutions of (1.4) in this paper. It is easy to see that if we know a parametric representation of $\varphi(\xi)$, then we have the solutions

$$
\int \varphi(\xi) d \xi=\Psi(\xi)=u(\xi),
$$

of (1.4). This paper is organized as follows. In section 2, in parametric space $\Omega=\left\{(k, r, c, \alpha) \mid(k, r, c, \alpha) \in R^{4}\right\}$, using the information of the phase portraits $\mathrm{in}^{[7]}$, we analyze all the possible travel wave solutions of the system (1.4), which correspond to periodic travel wave solutions of the system (1.1). Some explicit parametric representations of traveling wave solutions of (1.4) are obtained. The final section includes brief summary, future plans and potential fields of applications.

\section{Exact traveling wave Solutions of the system}

Lemma 1 When conditions $(A I)$ or $(A I I)$ hold, we can obtain some representative smooth exact periodic wave solutions of the system (1.6) as follows (see Fig1(1,2,3)):

(1) In Fig2 $(6,7,8)^{[7]},(1.6)$ has an exact periodic wave solution

$$
\varphi_{1}(\xi)=m_{1}+\frac{\left(m_{2}-m_{1}\right) s n^{2}\left(\omega \xi, g_{c}\right)}{1-\beta_{c}^{2} s n^{2}\left(\omega \xi, g_{c}\right)}
$$

Where 


$$
\begin{aligned}
& m_{1}<m_{2}<0<m_{3}<m_{4}, \beta_{c}^{2}=\left(\frac{m_{3}-m_{2}}{m_{3}-m_{1}}\right), \omega=\frac{\sqrt{\left(m_{2}-m_{1}\right)\left(m_{4}-m_{3}\right)}}{2}, \\
& g_{c}=\sqrt{\frac{\left(m_{3}-m_{2}\right)\left(m_{4}-m_{1}\right)}{\left(m_{4}-m_{2}\right)\left(m_{3}-m_{1}\right)}} .
\end{aligned}
$$

(2) In Fig2 $(2,3,4)^{[7]}$, (1.6) has a periodic wave solutions

$$
\varphi_{2}(\xi)=m_{4}+\frac{\left(m_{1}-m_{4}\right) \beta_{l}^{2} s n^{2}\left(\omega \xi, g_{l}\right)}{1+\beta_{l}^{2} \operatorname{sn}^{2}\left(\omega \xi, g_{l}\right)},
$$

where. $\quad m_{1}<m_{2}<m_{3}<0<m_{4} \quad, \quad \beta_{l}^{2}=\left(\frac{m_{3}-m_{4}}{m_{1}-m_{3}}\right)$, $g_{l}=\sqrt{\frac{\left(m_{1}-m_{2}\right)\left(m_{3}-m_{4}\right)}{\left(m_{1}-m_{3}\right)\left(m_{2}-m_{4}\right)}}$.

(3) In Fig2 $(10,11,12)^{[7]}$, (1.6) has an exact periodic wave solution

$$
\varphi_{3}(\xi)=m_{2}+\frac{\left(m_{2}-m_{3}\right) \beta_{r}^{2} s n^{2}\left(\omega \xi, g_{r}\right)}{1+\beta_{r}^{2} s^{2}\left(\omega \xi, g_{r}\right)},
$$

where $\quad m_{1}<0<m_{2}<m_{3}<m_{4} \quad, \quad \beta_{r}^{2}=\left(\frac{m_{1}-m_{2}}{m_{1}-m_{3}}\right)$, $g_{r}=\sqrt{\frac{\left(m_{1}-m_{2}\right)\left(m_{3}-m_{4}\right)}{\left(m_{1}-m_{3}\right)\left(m_{2}-m_{4}\right)}}$.

Theorem 1 From lemma1, three exact unbounded traveling wave solutions (see Fig1 $(4,5,6)$ ) of $(1.6)$ corresponding to the periodic wave solutions of (1.6) are obtained as follows:

$$
\begin{gathered}
u_{1}(\xi)=m_{1} \xi+\left(m_{2}-m_{1}\right) \int \frac{s n^{2}\left(\omega_{1} \xi, g_{c}\right)}{1-\beta_{c}^{2} \operatorname{sn}^{2}\left(\omega \xi, g_{c}\right)} d \xi, \\
u_{2}(\xi)=m_{4} \xi+\left(m_{1}-m_{4}\right) \beta_{l}^{2} \int \frac{s n^{2}\left(\omega \xi, g_{l}\right)}{1+\beta_{l}^{2} s n^{2}\left(\omega \xi, g_{l}\right)},
\end{gathered}
$$




$$
u_{3}(\xi)=m_{2} \xi+\left(m_{2}-m_{3}\right) \beta_{r}^{2} \int \frac{s n^{2}\left(\omega \xi, g_{r}\right)}{1+\beta_{r}^{2} s n^{2}\left(\omega \xi, g_{r}\right)} d \xi
$$

\section{Proof:}

By lemma1and (1.3), theorem 1 are easily proved.

Fig1:The unbounded wave solutions of the system (1.4) corresponding to the periodic wave solutions of the system (1.6).

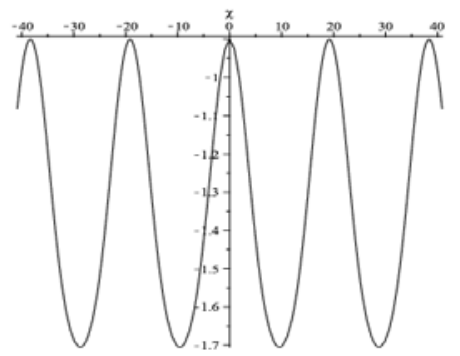

(1) $\varphi_{1}$

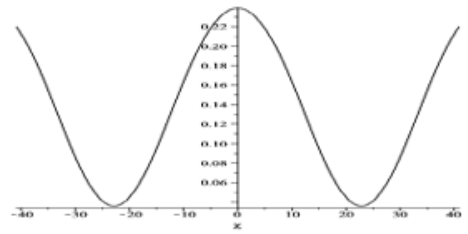

(3) $\varphi_{3}$

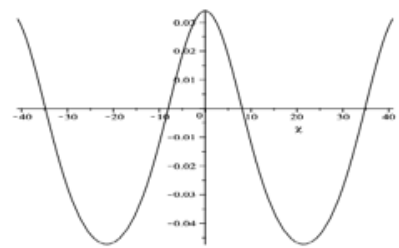

(2) $\varphi_{2}$

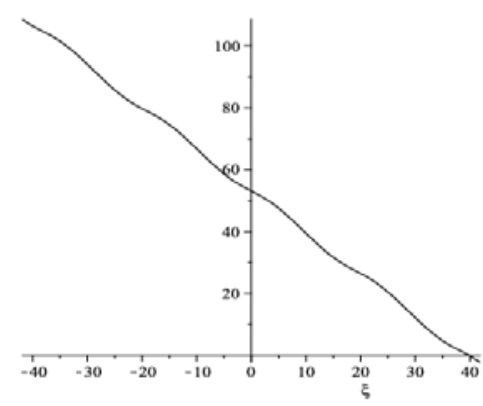

(4) $u_{1}$ 


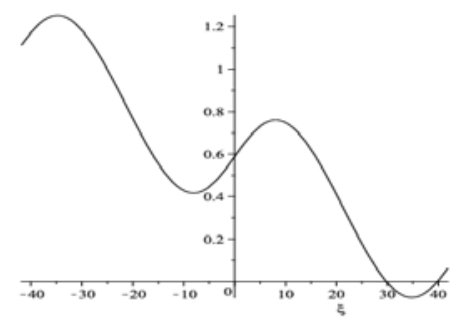

(5) $u_{2}$

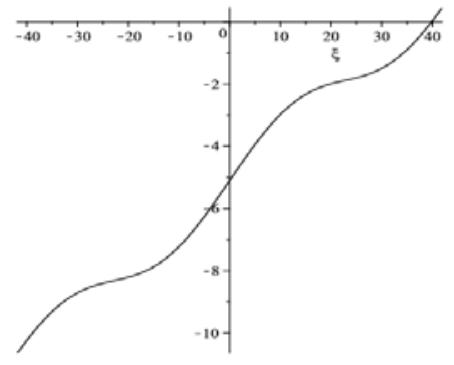

(6) $u_{6}$

\section{Conclusions}

In this paper, we discover that integration of all periodic wave solutions will be unbounded wave solutions. The unbounded wave solutions of (1.4) is fluctuating downward or upward (see Fig1(4-6)), which is polarization of motion. Our results are very helpful to analyze the character of traveling wave solutions.

\section{References}

[1] A. M. Wazwaz, Multiple soliton solutions for some (3+1)-dimensional nonlinear models generated by the Jaulent-Miodek hierarchy, Appl. Math. Lett. 21 (2012), no. 25, 1936--1940.

[2] A. M. Wazwaz, Partial Differential Equations and Solitary Waves Theorem, Springer and HEP, Berlin, 2009a.

[3] X. Geng, C. Cao and H.H. Dai, Quasi-periodic solutions for some (2+1)-dimensional integrable models generated by the Jaulent-Miodek hierarchy, 
J. Phys. A Math. Gen.12 (2001) no. 34, 989-1004.

[4] W. X. Ma, T. Huang and Y. Zhang, A multiple exp-function method for nonlinear

differential equations and its application, Phys.Scr. 21 (2010), no. 82, 065003.

[5] J. B. Li, Y. Zhang, X. H. Zhao, On a Class of Singular Nonliner Traveling Wave Equations (II): An Example of Gckdv Equations, Internat. J. Bifur. Chaos Appl.Sci. Engrg. 54 (2009), no. 19, 1995-2007.

[6] J. B. Li and G. R. Chen, Exact Traveling wave solutions and their bifurcations for the Kudryashov-Sinelshchikov equation, Internat. J. Bifur. Chaos Appl. Sci. Engrg.12 (2012), no. 22(5), 1250118-1-19.

[7] Y. P. Ran and J. Li, Bifurcation Method to Analysis of Traveling Wave Solutions for $(3+1)$-Dimensional Nonlinear Models Generated by the Jaulent-Miodek Hierarchy,The Bulletin of the Iranian Mathematical Society. (2014), in press.

[8] R. Hirota, The Direct Method in Soliton Theory, Cambridge Univ. Press, Cambridge,2004. 